\title{
Particle Tracking in Open Simulation Laboratories
}

\author{
Kalin Kanov \\ Department of Computer \\ Science \\ IDIES \\ Johns Hopkins University \\ Baltimore, MD 21218 \\ kalin@cs.jhu.edu
}

\author{
Randal Burns \\ Department of Computer \\ Science \\ IDIES \\ Johns Hopkins University \\ Baltimore, MD 21218 \\ randal@cs.jhu.edu
}

\begin{abstract}
Particle tracking along streamlines and pathlines is a common scientific analysis technique, which has demanding data, computation and communication requirements. It has been studied in the context of high-performance computing due to the difficulty in its efficient parallelization and its high demands on communication and computational load. In this paper, we study efficient evaluation methods for particle tracking in open simulation laboratories. Simulation laboratories have a fundamentally different architecture from today's supercomputers and provide publicly-available analysis functionality. We focus on the I/O demands of particle tracking for numerical simulation datasets 100 s of TBs in size. We compare data-parallel and task-parallel approaches for the advection of particles and show scalability results on data-intensive workloads from a live production environment. We have developed particle tracking capabilities for the Johns Hopkins Turbulence Databases, which store computational fluid dynamics simulation data, including forced isotropic turbulence, magnetohydrodynamics, channel flow turbulence and homogeneous buoyancy-driven turbulence.
\end{abstract}

\section{Categories and Subject Descriptors}

H.2.8 [Database Management]: Database Applications - Scientific Databases; H.2.4 [Database Management]: Systems - Distributed Databases; J.2 [Computer Applications]: Physical Sciences and Engineering - Physics

\section{Keywords}

scientific databases, data-intensive computing, particle tracking, turbulence

\section{INTRODUCTION}

Particle tracking is a powerful technique used for the analysis and visualization of flow fields and is an important tool in the study of fluid dynamics. It has been used to study Lagrangian velocity structure functions and tensor-based La-

Permission to make digital or hard copies of all or part of this work for personal or classroom use is granted without fee provided that copies are not made or distributed for profit or commercial advantage and that copies bear this notice and the full citation on the first page. Copyrights for components of this work owned by others than ACM must be honored. Abstracting with credit is permitted. To copy otherwise, or republish, to post on servers or to redistribute to lists, requires prior specific permission and/or a fee. Request permissions from permissions@ acm.org.

SC '15, November 15-20, 2015, Austin, TX, USA

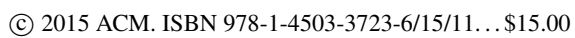
DOI: http://dx.doi.org/10.1145/2807591.2807645 grangian time correlation functions [29]. It has been used to generalize previous models of turbulent dispersion [3]. It has been used to explore the breaking of time-reversal-symmetry in turbulence 12. Many other examples are readily available. Particle tracing in scalar and vector fields and the visualization of such traces has been extensively studied in the context of supercomputing environments with a focus on the development of efficient parallelization techniques, which reduce the communication and computational load of the task $23,21,14$. The emergence of scientific analysis clusters for the long-term storage and curation of simulation datasets has introduced the need to revisit and explore evaluation strategies for particle advection in these markedly different environments. In this paper, we study efficient evaluation techniques for the tracing of particles in an open numerical simulation laboratory with a focus on the I/O demands of the task. The analysis cluster environment used in this study has a fundamentally different architecture than a highperformance computing (HPC) environment or a supercomputer. It consists of a distributed relational database cluster and publicly accessible Web services. It does not have the parallelism available in a supercomputing environment, but instead achieves high aggregate throughput based on I/O and network bandwidth. The numerical simulation datasets stored in the analysis database cluster are much larger than the amount of distributed memory available and therefore I/O becomes the main consideration as opposed to communication and computation.

Open simulation laboratories, such as the Johns Hopkins Turbulence Databases (JHTDB), aim to provide public access to world-class numerical simulation datasets as well as built-in analysis functionality, implemented following the "move the computation to the data" paradigm 10. It has already been shown that performing the analysis on the database nodes where the data are stored is much more efficient than transferring the data over the network to the user 20 and particle tracking is no exception. However, in the case of particle tracking the data requirements are not static and depend on the positions computed from each preceding iteration. This means that the data necessary to compute particle positions may reside on different database nodes during the advection process. Therefore, when particles cross the data boundary of a database node they either need to be reassigned or the data need to be retrieved from adjacent nodes. A straightforward approach that synchronizes the execution at a mediator level avoids this issue by redistributing particles to database nodes during every step of the process. However, this results in the sometimes un- 


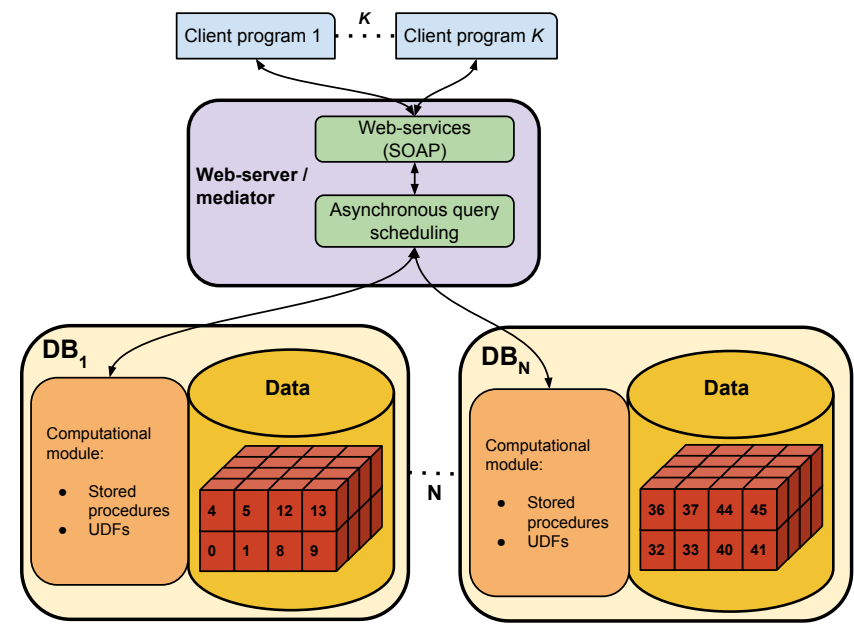

Figure 1: Architecture of the JHTDB.

necessary movement and reassignment of particles between the mediator and the database nodes. Such an approach also does not allow for asynchronous processing as particles are advected together in a batch and there is effectively a barrier after each iteration step.

Particle tracking functionality was first implemented in the JHTDB in 2011 and there have been nearly 6 million particle tracking requests made to the service since then. A total of 12.7 billion particles have been advected through the stored simulated flows with an average request size of $\sim 2200$ particles. This functionality has facilitated a number of research tasks, which have resulted in numerous publications, including research on turbulent dispersion 6, 3, 5], shape evolution [12], particles-turbulence interactions [7], calibration of experimental techniques 24 and others. The initial implementation adopted the straightforward approach of synchronizing particles after every iteration step at the mediator level, redistributing them to the database nodes, where the data are retrieved and next particle positions computed. The advantage of this approach is that particles are evaluated on each database node as a batch and the data for all particles are retrieved using a single database query. No data need to be retrieved from adjacent nodes and particle positions are evaluated using an efficient I/O streaming approach 13. Nevertheless, this approach has drawbacks as well, the majority of particles may not need to be reassigned to a different database node after each iteration step, which results in unnecessary communication between the mediator and the database nodes.

Previously, particle tracking was studied as a technique for the analysis and visualization of scalar or vector fields in scientific data on HPC architectures. Existing approaches rely on the massive parallelism available in supercomputers and in many cases avoid the I/O bottleneck by reading the entire dataset into memory 14, 21. In contrast, the amount of parallelism in analysis cluster environments is much smaller and the datasets are much larger than the distributed memory available. Additionally, scientists are interested in immersing particles and examining their evolution with the flow at different locations and time steps for analysis purposes and often require very accurate results. These analyses rely on accurate spatial and temporal interpolation routines, which are included in our implementations. Preprocessing the entire datasets and approximation techniques used for visualization 30] are therefore often not an option.

We study particle tracking for the purposes of scientific analysis in open simulation laboratories. We examine different approaches to the problem with a focus on the I/O requirements of the task. Our implementation provides particle tracking capabilities to scientists around the world that may not have access to supercomputers or the necessary sophistication to develop and run codes. The advection of particles is done on the database nodes where the data are stored. We show that a task-parallel implementation, which evaluates particles in batches using an I/O streaming method for the retrieval of data and evaluation of particle positions is up to 3 times faster than data-parallel asynchronous approaches or approaches that synchronize particle advection at a mediator level. We evaluate these approaches on microbenchmarks and user workload from the usage logs of the JHTDB.

The contributions of this paper are the following:

- A comparative study of particle tracking techniques in an open simulation laboratory, with a focus on the I/O demands of particle advection in simulation datasets up to 100 s of TB in size.

- A task-parallel method for the advection of particles that improves performance by up to a factor of 3 and achieves scalability by performing the entire process on the database nodes of the analysis cluster environment.

- An evaluation of the trade-offs between batch execution and asynchronous processing, which shows that the reduced I/O time from the retrieval of data for an entire batch of particles by means of an I/O stream outweighs the advantages of asynchronous processing, as asynchronous execution results in an increased number of smaller-sized I/O requests.

\section{BACKGROUND}

\subsection{Johns Hopkins Turbulence Databases}

The Johns Hopkins Turbulence Databases (JHTDB) 16 20 is an open simulation laboratory for the study of fluid dynamics. It provides public access to world-class numerical simulation datasets of turbulence to anyone in the world with an Internet connection. In addition to providing access to the data, the JHTDB also implements a number of core scientific analysis techniques, which are executed in the analysis cluster environment, where the data are stored. It currently stores four datasets, the output of a forced isotropic turbulence simulation, the output of a magnetohydrodynamics simulation, the output of a channel flow simulation and the output of a homogeneous buoyancy-driven turbulence simulation. The total amount of data of these datasets is over 200 TB.

The JHTDB stores turbulence simulation data in a cluster of relational databases. The database nodes are part of the 1.1 PB capacity GrayWulf cluster 27] and the 11 PB capacity DataScope cluster 4 at Johns Hopkins. SQL Server was chosen as the backend database engine, because of its ability to handle large datasets efficiently, support for partitioned tables and custom partitioning functions, efficient data ingestion capabilities, integration with the Common Language 
Run-time (CLR) and support for user-defined functions and CLR stored procedures written in an object-oriented language. The JHTDB also builds on the success and experience of storing scientific data in a relational database cluster of the Sloan Digital Sky Survey 25].

The data reside on a three-dimensional spatial grid, which is $1024^{3}$ in size and consists of 1024 time steps for most of the datasets (the channel-flow dataset has a bigger grid and 4000 time steps). Each simulation time step is spatially subdivided into small blocks or atoms, which are of size $8^{3}$ data points. These atoms are distributed across the nodes of the cluster. The Morton order Z-curve governs the spatial partitioning and distribution of the data 20]. Scientific analysis functionalities are implemented as user-defined functions or stored procedures in each database. The data and analysis functionality are accessible through Web services hosted on a Web server front-end (http://turbulence.pha.jhu.edu). The Web server also acts as a mediator, distributing user requests and queries to the database nodes and initiating their distributed evaluation. The architecture of the JHTDB is shown in Figure 1 .

The JHTDB implements a variety of different analysis tasks and scientific functions, not limited to particle tracking. The spatial partitioning and distribution of data have therefore been carefully chosen to provide the best I/O performance for a variety of analyses with different workloads, such as spatial differentiation, thresholding, vorticity studies and subgrid-scale modeling, to name a few. Most queries perform kernel computations, which require data in a neighboring region around a target location (this includes particle tracking). The kernels are usually of size $4^{3}$ to $8^{3}$ and the database atom size was chosen so that data for each individual computation are retrieved with a small number of I/Os. A previous version of the database 20] used larger atoms $\left(64^{3}\right)$. We chose the smaller atoms because the larger atoms resulted in the retrieval of more than the necessary data for a computation. In doing so, we evaluated many different sizes and found that the I/O performance of smaller atoms outperforms that of larger atoms 13. All database atoms for a batch query are retrieved by means of a join and the database can efficiently process this join by making read-ahead and prefetching decisions.

\subsection{Particle Tracking}

Particle tracking is implemented in the JHTDB by means of a GetPosition function 29]. The function tracks a list of particles simultaneously and returns the final particle locations at the end of the trajectory integration time specified by the user. Particle tracking is performed using a second order Runge-Kutta integration scheme. This scheme was chosen because coupled with high order spatial and temporal interpolation (Section 2.3 it provides sufficient accuracy to perform scientific analysis while having lower I/O demands than a higher order integration scheme. A detailed study of the accuracy of the particle tracking implementation in the JHTDB is presented by $\mathrm{Yu}$ et al. 29. We briefly describe the routine below.

The GetPosition function takes as parameters a list of particle locations $\mathbf{y}$, a start time $\left(t_{S T}\right)$, an end time $\left(t_{E T}\right)$ and a particle integration time step $\left(\Delta t_{p}\right)$ and returns the particle locations at the specified end time. Fluid particles can be tracked both forward in time by specifying $t_{E T}>t_{S T}$ and backward in time by specifying $t_{E T}<t_{S T}$. Particle tracking is accomplished by integrating between times $t_{S T}$ and $t_{E T}$ the equation

$$
\frac{\partial \mathbf{x}^{+}(\mathbf{y}, t)}{\partial t}=\mathbf{u}^{+}(\mathbf{y}, t)
$$

where $\mathbf{x}^{+}(\mathbf{y}, t)$ and $\mathbf{u}^{+}(\mathbf{y}, t)$ denotes the position and velocity at time $t$ of the fluid particle originating from position $\mathbf{y}$ at initial time $t_{S T}$ (superscript + represents Lagrangian quantities following the fluid particle). We replace the Lagrangian velocity $\mathbf{u}^{+}(\mathbf{y}, t)$ with the Eulerian velocity from the database $\mathbf{u}(\mathbf{x}, t)$ at the particle location $\mathbf{u}^{+}(\mathbf{y}, t)=\mathbf{u}\left(\mathbf{x}^{+}(\mathbf{y}, t), t\right)$.

To advance the particle positions between two successive time instants $t_{m}$ and $t_{m+1}\left(=t_{m}+\Delta t_{p}\right)$ the second order Runge-Kutta integration scheme consists of two steps. The predictor step yields an estimate:

$$
\mathbf{x}^{*}=\mathbf{x}^{+}\left(\mathbf{y}, t_{m}\right)+\Delta t_{p} \mathbf{u}^{+}\left(\mathbf{y}, t_{m}\right) .
$$

The corrector step then computes the particle position at $t_{m+1}$ using the predictor estimate and the original particle location:

$$
\begin{aligned}
& \mathbf{x}^{+}\left(\mathbf{y}, t_{m+1}\right)=\mathbf{x}^{+}\left(\mathbf{y}, t_{m}\right)+ \\
& \Delta t_{p}\left[\mathbf{u}^{+}\left(\mathbf{y}, t_{m}\right)+\mathbf{u}^{+}\left(\mathbf{x}^{*}, t_{n+1}\right)\right] / 2 .
\end{aligned}
$$

In order for the integration to end exactly at the specified $t_{E T}$ the integration time step is equal to the smaller of the specified $\Delta t_{p}$ and the difference between $t_{E T}$ and $t_{m}$. Particle advection proceeds until $t_{m}$ reaches the user-specified final time $t_{E T}$. The GetPosition function then returns the final particle positions $\mathbf{x}^{+}\left(\mathbf{y}, t_{E T}\right)$ for all particles that were at initial locations $\mathbf{y}$.

\subsection{Spatial and Temporal Interpolation}

Accurate spatial and temporal interpolations are important to obtain the fluid velocities while tracking fluid particles. Spatial interpolation using Lagrange polynomials with various optional orders of accuracy can be specified by the user and temporal interpolation is done by default using piecewise cubic Hermite interpolation. We briefly describe these interpolation techniques and their data requirements here.

The interpolated value of a variable at a target location that does not coincide with a grid node can be computed using Lagrange polynomial interpolation. Lagrange polynomial interpolation operates over a cubic region surrounding the target location. This region is the kernel of computation and the data values at all grid locations within that region are needed for the computation of the interpolated value. The interpolated value of any point in space $\mathbf{x}^{\prime}$ computed using $N^{t h}$ order Lagrange polynomial interpolation is given by:

$$
\begin{gathered}
f\left(\mathbf{x}^{\prime}\right)=\sum_{k=1}^{N} \sum_{j=1}^{N} \sum_{i=1}^{N} l_{z}^{q-\frac{N}{2}+k}\left(z^{\prime}\right) l_{y}^{p-\frac{N}{2}+j}\left(y^{\prime}\right) l_{x}^{n-\frac{N}{2}+i}\left(x^{\prime}\right) \\
\cdot f\left(x_{n-\frac{N}{2}+i}, y_{p-\frac{N}{2}+j}, z_{q-\frac{N}{2}+k}\right),
\end{gathered}
$$

in which $\mathbf{x}^{\prime}=\left(x^{\prime}, y^{\prime}, z^{\prime}\right)$ is the position of the target point in 3-dimensional space and $f\left(x_{i}, y_{j}, z_{k}\right)$ represents the data stored at the node on the grid at location $\left(x_{i}, y_{j}, z_{k}\right)$. The computation kernel is an $N \times N \times N$ cube around the target point. The location $\left(x_{n}, y_{p}, z_{q}\right)$ on the grid is computed 
as $n=\left\lfloor\frac{x^{\prime}}{\Delta x}+\frac{1}{2}\right\rfloor, p=\left\lfloor\frac{y^{\prime}}{\Delta y}+\frac{1}{2}\right\rfloor, q=\left\lfloor\frac{z^{\prime}}{\Delta z}+\frac{1}{2}\right\rfloor$, where $\Delta x, \Delta y, \Delta z$ are the widths of the grid in the $x, y$ and $z$ dimensions. The Lagrange coefficients $l$ are given by:

$$
l_{\theta}^{i}\left(\theta^{\prime}\right)=\frac{\prod_{j=\alpha-\frac{N}{2}+1, j \neq i}^{\alpha+\frac{N}{2}}\left(\theta^{\prime}-\theta_{j}\right)}{\prod_{j=\alpha-\frac{N}{2}+1, j \neq i}^{\alpha+\frac{N}{2}}\left(\theta_{i}-\theta_{j}\right)},
$$

in which $\theta$ can be $x, y$ or $z$ and $\alpha$ can be $n, p$ or $q$, respectively.

Similarly to spatial interpolation, temporal interpolation can be used to obtain the value of a variable in-between stored time steps with higher accuracy. Temporal interpolation can be performed using Picewise Cubic Hermite Interpolation Polynomials (PCHIP). In the JHTDB, the value from the two nearest time steps is interpolated at time $t^{\prime}$ with centered finite difference evaluation of the end-point time derivatives. A total of four temporal points are used:

$$
\begin{gathered}
f\left(t^{\prime}\right)=a+b\left(t^{\prime}-t_{n}\right)+c\left(t^{\prime}-t_{n}\right)^{2}+ \\
d\left(t^{\prime}-t_{n}\right)^{2}\left(t^{\prime}-t_{n+1}\right),
\end{gathered}
$$

in which $f\left(t_{n}\right)$ denotes the data stored at time $t_{n}, n=$ $\left\lfloor\frac{t^{\prime}}{\Delta t}+\frac{1}{2}\right\rfloor$ with $\Delta t$ being the time increment between consecutive times stored in the database and

$$
\begin{gathered}
a=f\left(t_{n}\right) \\
b=\frac{f\left(t_{n+1}\right)-f\left(t_{n-1}\right)}{2 \Delta t} \\
c=\frac{f\left(t_{n+1}\right)-2 f\left(t_{n}\right)+f\left(t_{n-1}\right)}{2 \Delta t^{2}} \\
d=\frac{-f\left(t_{n-1}\right)+3 f\left(t_{n}\right)-3 f\left(t_{n+1}\right)+f\left(t_{n+2}\right)}{2 \Delta t^{3}} .
\end{gathered}
$$

In order to obtain accurate fluid velocities while performing particle tracking PCHIP interpolation is used by default in the JHTDB, which means that every step of the RungeKutta integration (both the predictor and corrector steps) requires a query to 4 time steps stored in the database. Spatial interpolation using Lagrange polynomials of 4 th, 6 th and 8 th order can be specified by the user. This means that every particle's position is computed using 64, 216 or 512 data points, respectively.

\subsection{Scientific Use Cases}

The ability to track particles both forward and backward in time, without having to retrieve any data to a local machine or handling the distribution of particles and their advection has allowed scientists from around the world to perform sophisticated analysis tasks using their desktops or laptops. Providing this capability for landmark turbulence simulation datasets and making it publicly available has enabled new insights in the study of turbulence. As an example, analysis of this type has revealed why the breaking and reconnection of magnetic field-lines in solar flares lasts several minutes and not millions of years as predicted by classical theory 6.

Fluid particle tracking is one of the most important techniques in the study of the Lagrangian description of tur- bulence, in which a large number of particle trajectories are followed in order to capture the overall space and timescales of the flow. Studies of turbulent transport and mixing processes often make use of the Lagrangian description of turbulence. Other examples include studying entrainment processes at turbulent/laminar interfaces [11 and modeling processes such as atmospheric pollution transport and turbulent combustion 22. Extracting Lagrangian data from stored numerical simulation data requires an iterative process, which queries multiple time steps of the simulation. This process is complicated further by the fact that particles' spatial locations are continuously changing. In a distributed environment their subsequent evaluation may require data stored on different database nodes. The GetPosition functionality of the JHTDB is a valuable tool, which facilitates Lagrangian analysis and here we study efficient approaches for its implementation.

\section{PARTICLE TRACKING METHODS}

We describe three approaches to the implementation of particle tracking in open simulation laboratories. These approaches share some common aspects with techniques used for the visualization of particle traces in high-performance computing environments or supercomputers. We implement these approaches in a markedly different environment, a scientific analysis cluster consisting of a sharded relational database cluster, which stores the numerical simulation data and a Web server front-end, which hosts publicly-available Web services and acts as a mediator. The particle tracking functionality is moreover used not for visualization, but for analysis tasks and requires accurate spatial and temporal interpolation for the computation of each particle position.

The three approaches that we examine are a task-parallel method, a data-parallel method and a method which synchronizes execution at the Web server/mediator level. The task-parallel approach distributes particles based on the spatial partitioning of the data, but allows them to be integrated forward on a database node even if they cross the node's data boundary. The data-parallel approach implements services running on each database node and integrates particles forward only if they are within the node's data boundary. When a particle crosses the data boundary it is reassigned to the appropriate node or nodes for evaluation. This method supports asynchronous processing of individual particles and queries data from multiple time steps as needed. Finally, the last method which we examine is a straightforward approach to particle tracking, which synchronizes the evaluation at the mediator level. This is also the method that was initially implemented in the JHTDB.

\subsection{Task-parallel Evaluation}

The task-parallel approach to particle tracking effectively deals with the data dependency that arises during particle advection. Namely, the fact that each subsequent integration step during the advection process depends on the results of the previous step. This approach distributes the initial particle positions to the database nodes based on the spatial partitioning of the data and each database process performs the particle advection for the entire batch of particles assigned to it until completion (Fig. 2). No reassignment of particles is necessary and the fact that particles are advected together as a batch means that the data retrieved from the database is for the same set of time steps during each in- 


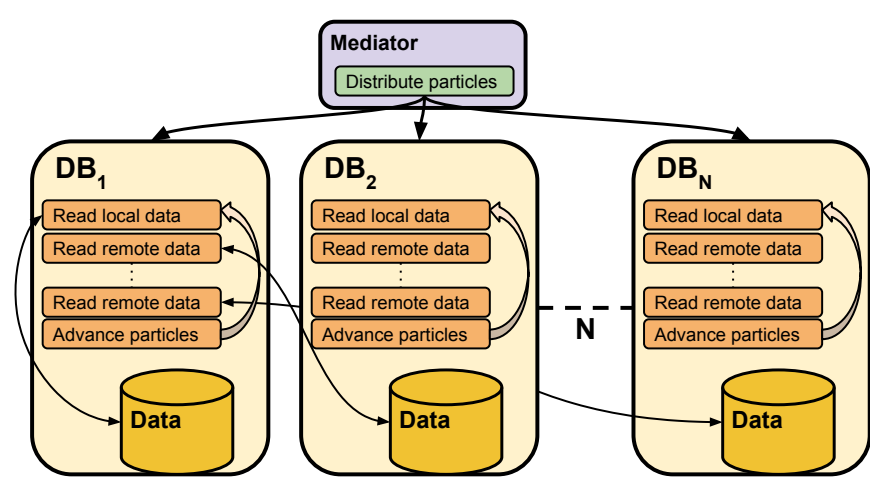

Figure 2: Task-parallel particle tracking approach.

tegration step. This allows us to make full use of the $\mathrm{I} / \mathrm{O}$ streaming method for the evaluation of batch queries performing spatial and temporal interpolation 13 . Using this method particle positions are evaluated by means of partialsums, which allows us to perform a single sequential read over the data.

The overall execution strategy proceeds as follows. The initial particle locations are preprocessed into a collection of key-value dictionaries. The keys correspond to the indexes of the database atoms needed for the advection of a particle. The values stored in the dictionaries are lists of particles, where each particle in a list needs data from the database atom with the corresponding key. There is one such dictionary per database node. We create a temporary table out of the dictionary keys on each database node that stores data required for the advection of particles. The data atoms with the corresponding indexes are then retrieved using a join between this temporary table and the table storing the simulation data. As a database atom is retrieved from the database, it is routed to each particle that needs data from it and the particle's interpolated velocity is updated with the partial-sum computation over the overlapping region between the database atom and the particle's kernel of computation. Once all database atoms associated with a particle are retrieved, we compute the next particle position based on the computed velocity. When all particles' next positions are evaluated the dictionaries are cleared and repopulated based on the new particle positions. The process continues until all particles reach the specified end time.

The task-parallel approach must handle particles that have crossed the data boundary of a database node. Each process maintains a connection to all database nodes, which have data necessary for the evaluation of any given particle's position. A connection is opened only if there are particles that need data from the particular database and the data for all particles that have partially or fully crossed into a different database are retrieved as a batch. The I/O streaming method supports distributed evaluation, which allows us to evaluate particle positions even if the data are coming from different databases. Given that the data are distributed across a relatively small number of nodes (4 to 8 ) and the spatial volume of a dataset is large $\left(1024^{3}\right.$ data points) the number of particles that cross into a different database is not large. Moreover, the scientific cluster environments are built with the goal of providing network speeds that are onpar if not faster than the aggregate disk read speeds, which

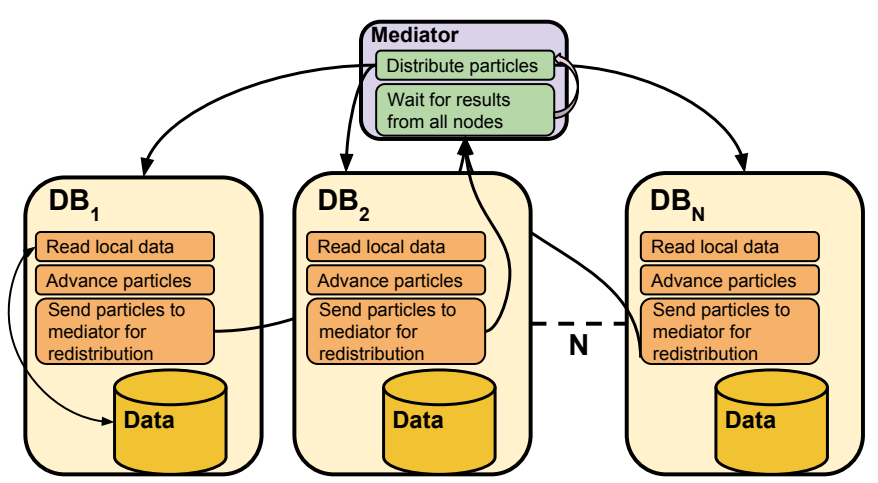

Figure 3: Synchronization of particle integration at the mediator.

means that there is little downside to the retrieval of data from adjacent database nodes.

The use of the I/O streaming method for the evaluation of particle positions during each integration step and the evaluation of all particles as a batch minimizes the I/O incurred during each step. The method retrieves only the database atoms that cover the kernels of computation associated with each particle. It schedules the retrieval of all database atoms required by the entire batch of particles by means of a single join query, which allows the database to pick the most efficient strategy for the execution of the query and make use of read-ahead and prefetching. The same strategy applies when retrieving data from adjacent nodes. We retrieve only the database atoms required, but all of them are retrieved by means of a single query.

The use of static initial distribution of particles with the task-parallel approach aims to balance the execution around $\mathrm{I} / \mathrm{O}$. We have found that this has the biggest impact on overall execution time of particle tracking queries. Nevertheless, it is straightforward to modify this approach with the goal of balancing the computational load on each database node, by distributing equal-sized particle batches to each database node.

\subsection{Data-parallel Evaluation}

The data-parallel particle tracking approach distributes particles to processes based on the spatial partitioning of the data. Each process advects particles, for which the necessary data are available locally and reassigns particles to other nodes if they cross the data boundary. This approach allows for the asynchronous movement and reassignment of particles from process to process. Each process has to retrieve data from multiple different time steps depending on the progress of the particles assigned to it at any given step. This approach is based on the established parallelization paradigm of static allocation of blocks to processes 23. The difference however is that instead of reading all blocks assigned to a process into memory at the start of the computation, the data necessary for the evaluation of all particles assigned to a process are continuously read from the local database.

The data-parallel approach performs particle tracking as follows. The mediator distributes the particles to processes running on each database node based on the static spatialpartitioning of the data. The processes are implemented as services in the Windows Communication Framework (WCF). 
Each process then begins advecting particles by retrieving all of the data needed for the computation of the positions of the entire batch of particles. Each process keeps track of whether a particle has crossed the local data boundary or whether a particle has reached the end time and its advection is therefore complete. Particles that have crossed the local data boundary are reassigned to the appropriate process by means of a one-way send. Particles that have partially crossed a data boundary are assigned to multiple processes for evaluation and the partial results are sent back to the process that owns the particle. Finally, when a particle has reached the specified end time it is sent back to the mediator. When the mediator receives all particles it sends the final positions back to the user.

One of the drawbacks of the data-parallel approach is that because of the asynchronous nature of the movement of particles between processes, each process has to retrieve data from multiple different time steps at any given time. This increases the overall I/O and subsequently execution time. Additionally, for workloads that result in a large number of particle crossings, the movement and reassignment of particles increases the communication costs. Recently, domain traversal approaches that make use of data-parallelism have shifted towards group-based communication flows in order to alleviate some of these issues. For example, the DStep system [14 makes use of worker groups with an assigned communicator, which batches communication requests and executes them in a group-based manner.

\subsection{Synchronization at Mediator Level}

Similarly to the data-parallel particle tracking approach, the last method that we examine distributes and advects particles only to processes that have the data necessary for each integration step available locally. However, this approach achieves this goal by different means. In order to support the batch execution of particles it sends particle positions back to the mediator after each integration step and the particles are then redistributed based on their new positions. This mode of execution effectively places a barrier after each integration step, which guarantees that each process has all of the data necessary for particle advection available locally and can retrieve them by means of a single sequential read. Each process computes the particle velocities and the new particle positions using the I/O streaming method for the execution of batch queries 13. The process is shown in Figure 3 .

This is a straightforward approach to particle tracking and this is the method that was initially implemented in the JHTDB for the GetPosition function. This method guarantees optimal I/O for each integration step. However, this comes at the cost of increased communication between the mediator and the database processes performing the integration. All particles are sent back to the mediator after each integration step even though the majority of them may not have crossed the data boundaries of the process they are assigned to and therefore do not need to be reassigned. Additionally, the mediator synchronization means that particle advection proceeds at the pace of the slowest process.

\section{EXPERIMENTAL RESULTS}

We evaluate the performance of the different particle tracking approaches in the context of the JHTDB and more broadly in scientific analysis cluster environments, which store large

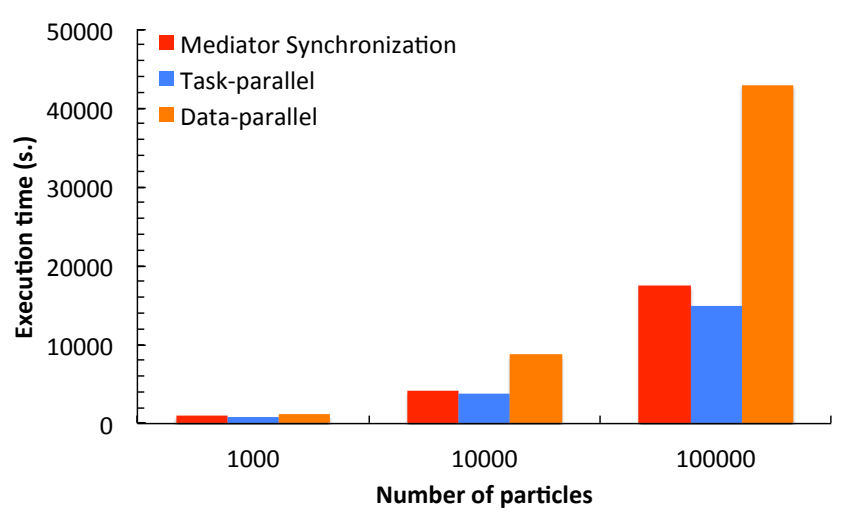

Figure 4: Execution times of the different particle tracking approaches for particles randomly distributed in the entire data volume with an integration step less than half of the temporal resolution of the data.

numerical simulation datasets. The evaluation is carried out from the point of view of the end user and includes all aspects of the process, from the initial request, to particle distribution, the I/O performed at each integration step, the integration time and finally the time to send the results back to the user. For the performance evaluation we use the datasets available in the JHTDB. Most of the experiments are run against the magnetohydrodynamics (MHD) dataset, which has 1024 time steps and stores data on a $1024^{3}$ spatial grid [1]. Additionally, we evaluate the different approaches on a forced isotropic turbulence dataset [16] and on a homogeneous buoyancy-driven turbulence (HBDT) dataset 17.

We perform micro-benchmarks designed to examine the different characteristics of the particle tracking approaches and to offer a study at large scale, including large number of particles to be traced and long time spans. We also evaluate the approaches on workload extracted from the usage log of the JHTDB. All experiments were carried out on the live production database nodes of the JHTDB through a development Web server/mediator hosting the Web services. The system and the database cluster were therefore processing workload from regular users during the experimental runs.

\subsection{Experimental Setup}

The nodes of the database cluster are part of the GrayWulf 27] and DataScope 4] clusters at JHU. The GrayWulf database nodes are $2.66 \mathrm{GHz}$ dual quad-core Windows 2008 servers with SQL Server 2008 R2 and 24 GB of memory. Each node has 24 2TB SATA disks arranged as a set of four RAID-5 arrays. The DataScope database nodes are 3.47 GHz dual quad-core Windows 2008 servers with SQL Server 2008 R2 and 48 GB of memory. Each node has 242 TB SATA disks arranged as 12 mirrored disk arrays. The MHD dataset is partitioned spatially across 4 GrayWulf nodes with one database per node and database files striped across the nodes and their associated disk arrays. The forced isotropic turbulence dataset is also partitioned across 4 GrayWulf nodes, but in this case there are 2 databases per node. The HBDT dataset is partitioned across 2 DataScope nodes with 4 databases per node. 


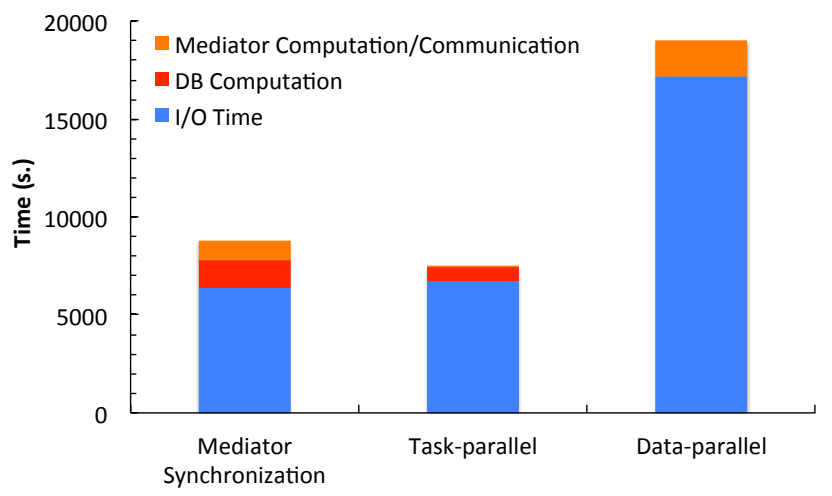

Figure 5: Breakdown of the execution time of the different particle tracking methods.

\subsection{Micro-benchmarks}

The first set of experiments that we perform evaluate the different approaches on workloads of varying number of particles, tracking them over a relatively large time span. Figure 4 shows the execution time for particle batches of 1000 , 10,000 and 100,000 in the MHD dataset. For this set of experiments we run a single process per database node. The time span of each request is a fifth to a quarter of the entire time span of the simulation. This is a much larger time span than what is observed in typical user workload, but it allows particles to travel a large distance and can be used to examine the overall structure of the flow. Particles are distributed randomly in the entire data volume and an integral time step of about one half to one third of the simulation time step is used, which is the resolution at which user workloads typically operate. This results in a total of $\sim 1000$ steps in the Runge-Kutta integration scheme, which are carried out over 200 simulation time steps. Each particle position is computed using 4th order Lagrange polynomial interpolation, which is again typical of the observed workload. Temporal interpolation is used by default, using data from 4 time steps to evaluate every particle position.

As we can see from the results in the figure, the taskparallel approach outperforms the other two approaches by at least $\sim 15 \%$. This is because it achieves a good balance between the retrieval of data for the entire batch of particles assigned to a process and allowing each process to advect particles independently. The data-parallel approach performs significantly worse. This is mainly due to the asynchronous nature of the particle tracking process in this case, which forces each process to retrieve data from multiple time steps in order to advect the particles assigned to it during each integration step. Additionally, because of the lack of ghost regions around data boundaries, particles that are near the boundary have to be assigned to multiple processes for evaluation. Replicating data around the boundary is not an option in the JHTDB because of the large overhead that this introduces and the fact that many analysis queries do not need it.

The algorithms spend most of their time performing the I/O necessary for particle advection. Figure 5 shows the time each method spends performing I/O relative to the overall execution time. For this experiment, we used a workload similar to the workloads presented in Figure 4 We

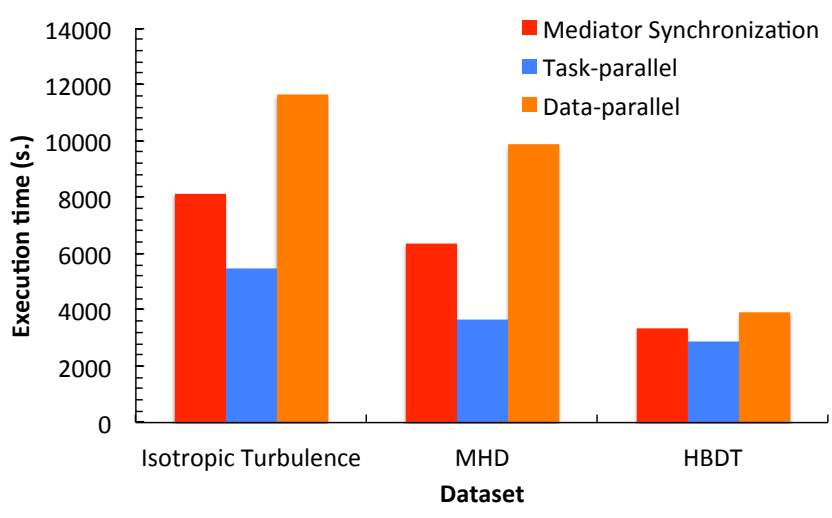

Figure 6: Execution times of the different particle tracking approaches for different workloads in three different datasets (forced isotropic turbulence, MHD and HBDT).

advected 100,000 particles randomly distributed in the entire data volume over $\sim 100$ simulation time steps with an integral time step of about one half to one third of the stored simulation time step. The task-parallel method spends nearly $90 \%$ of the time performing I/O. This I/O time is $\sim 6 \%$ higher than the I/O time for the method that performs synchronization at the mediator level. This is mainly due to the need to retrieve data from adjacent nodes. Synchronizing at the mediator leads to an optimal I/O plan for every Runge-Kutta integration step as no data need to be retrieved from adjacent nodes. However, this comes at the cost of increased communication with the mediator and increased pre-processing times for each stored procedure call in the database. The task-parallel method's mediator time to distribute the initial particle positions and to assemble the final results is negligible. On the other hand, the $\mathrm{I} / \mathrm{O}$ time of the data-parallel method is more than 2.5 times larger. This is due to the fundamentally different I/O plan induced by the asynchronous nature of the processing. Data are potentially retrieved from multiple different time steps during every integration step and the data retrieval requires different query plans. The rest of the time is spent performing computation and coordinating the reassignment of particles between the processes.

The next set of experiments evaluates the three particle tracking approaches in different datasets, on different workloads and with different number of processes per database node (Fig. 6). The first dataset used in the experiments is a forced isotropic turbulence dataset, which is the result of a direct numerical simulation (DNS) of stationary hydrodynamic turbulent flow based on a solution to the NavierStokes equations using a pseudo-spectral parallel code. The second dataset is a forced magnetohydrodynamics turbulence dataset, which is the result of a DNS of the incompressible MHD equations using a pseudo-spectral parallel code with a Taylor-Green flow as the external stirring force. The third and last dataset is a homogeneous buoyancy driven turbulence dataset, which is the result of a DNS solving the miscible two-fluid incompressible Navier-Stokes equations.

The number of processes varies mainly because of the different spatial distribution of the data for the different datasets. The workload for the forced isotropic turbulence 


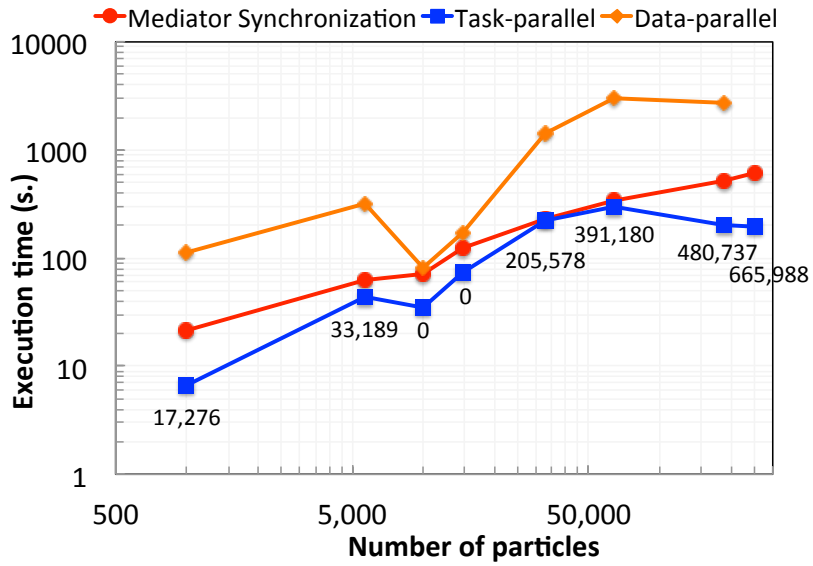

Figure 7: Performance of the different particle tracking approaches on workload extracted from the usage log of the JHTDB. Labels show the number of data boundary crossings.

dataset consists of 100,000 particles with an integration step of around one third the simulation time step and traces particles over a time span of about a fifth of the entire time span stored in the database. Similarly as for the MHD dataset this results in a total of $\sim 1000$ steps in the RungeKutta integration scheme. This dataset is distributed across 4 database nodes with 2 databases per node. We use 4 processes per database, for a total of 32 processes in these experiments. The workload for the MHD dataset is the 100,000 particle workload shown in Figure 4 but in this case we use 8 processes per database node, for a total of 32 processes. Finally, the workload for the HBDT dataset is 10,000 particles with similar integration step and time span as for the other two datasets. This dataset is distributed across 2 database nodes with 4 databases per node and we use 2 processes per database for the experiments.

The task-parallel approach performs best for all of the different datasets and parameterizations. In fact it is $75 \%$ faster than the mediator-synchronization approach for the MHD dataset and $50 \%$ faster than the mediator-synchronization approach for the forced isotropic turbulence dataset. This is due to the fact that the task-parallel approach is able to scale better with increased process count as particle advection is carried out by each database process until completion of the entire batch and there is no movement of particles between the mediator and the database processes. The communication between the mediator and the database processes after each integration step does not scale as well with increased process count.

The data-parallel approach's relative performance is significantly better for the HBDT dataset as compared to the other two datasets. It is only $36 \%$ slower than the taskparallel approach, where as for the MHD dataset it is 2.7 times slower. This is due to the smaller size of the workload, which leads to particles making substantially fewer data-boundary crossings. In this case, there were around 220,000 such crossings, while the total number of crossings for the MHD dataset was 2.9 million. Moreover, the higherend database nodes that host the HBDT dataset have faster CPUs, more memory and newer network cards.
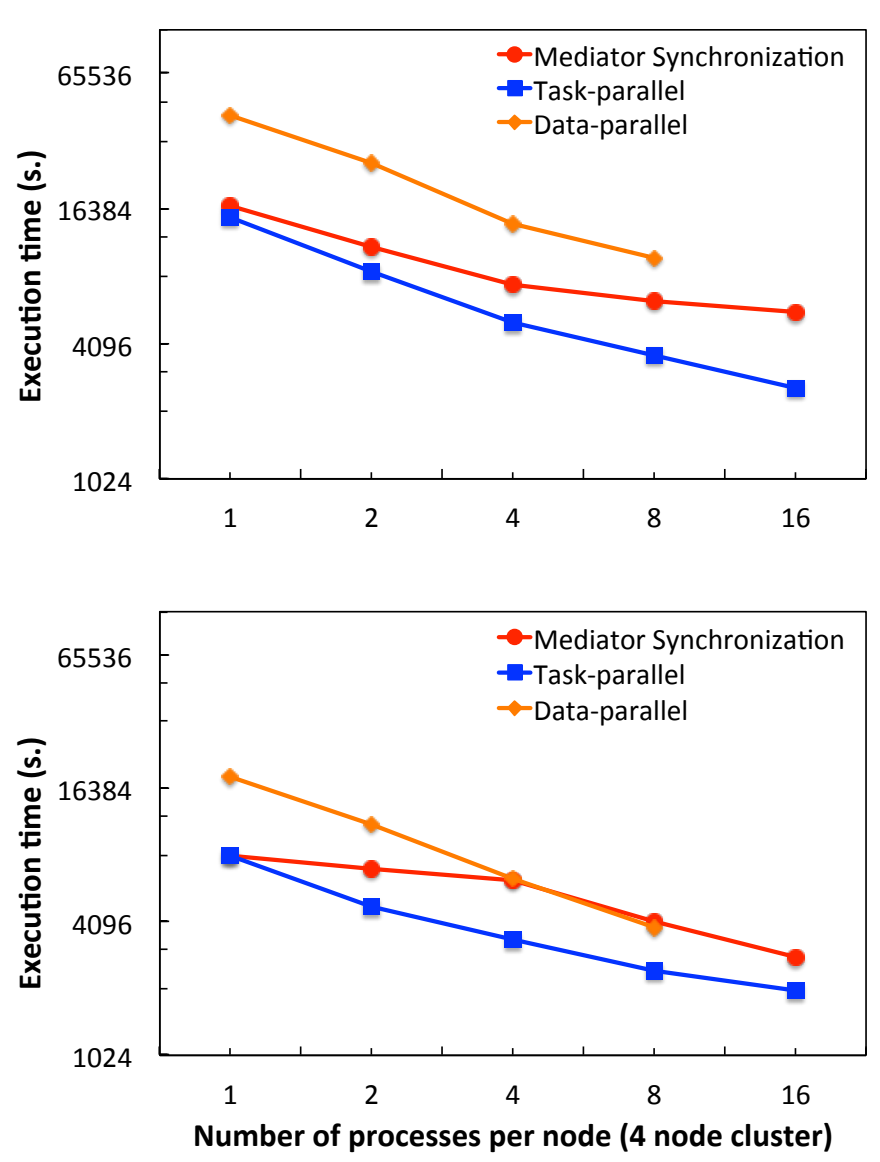

Figure 8: Performance of the different particle tracking approaches for 100,000 randomly distributed particles over 200 time steps (top panel) and for 10,000 randomly distributed particles over 500 time steps (bottom pannel) with different number of processes per database node in a 4 node cluster.

\subsection{User Workload}

We evaluate the different particle tracking approaches on workload extracted from the usage logs of the JHTDB. Our analysis of the usage logs shows that most of the users' workload requests particle positions integrated over a relatively short time span compared to the time span of the entire simulation. Users also request integration time steps that are a fraction of the separation between simulation time steps stored in the database. For the experiments we have selected from the most commonly executed particle tracking queries with varying number of particles, from 1,000 to 250,000. Particles were integrated for a total of 100 Runge-Kutta integration steps over 20 simulation time steps. The results are shown in Figure 7

Overall the task-parallel approach performed the best and was up to 3 times better than synchronization at the mediator. This performance is even better than what we measured for the micro-benchmarks. This is because most user workload tends to be more localized, for example examining a region of interest, and in such cases particles do not cross data boundaries very often and each process performs more 
localized I/O. For each run we measured the number of times a particle crossed the data boundaries of the process that it was assigned to (data points are labeled with these numbers in Figure 7).

User workloads distribute particles in the simulation domain with varying sparsity and localization characteristics. Some workloads distribute particles randomly or nearly randomly in the entire data volume as is the case for the 64,000 particles workload. Others confine particles to a much smaller region in space as is the case for the 185,000 and 250,000 particles workloads. The 10,000 and 14,641 particles workloads, which exhibit 0 particle crossings also distribute particles densely in space, bur moreover they happen to seed the initial particle positions near the center of the data region stored on a database node. Given the relatively short integration time, these "particle clouds" do not move far enough from the initial particle positions and do not cross into the data regions stored on adjacent nodes, resulting in the 0 crossings observed in these workloads.

While particle crossings have an impact on the overall execution time in some cases higher number of crossings does not necessarily lead to higher execution time. Another factor that has an impact on the execution time is the number of data blocks requested from the database. For example, the workload with 64,000 particles, which had 391,180 particle crossings had a higher execution time than the workloads with 185,000 and 250,000 particles. The 64,000 particle workload was distributing particles randomly in the entire data volume and subsequently almost all of the data blocks for each time step had to be retrieved during particle advection. On the other hand, the other two workloads were much more localized, resulting in requests for only about 1 to $2 \%$ of all data blocks of a time step. The task-parallel approach is effectively able to take advantage of these workload characteristics, which reduces the overall running time. Synchronization at the mediator is much less sensitive to data locality as particles are continuously sent back and forth for reassignment and the size of the request is the dominating factor.

\subsection{Scalability}

Particle tracking for scientific analysis is a data-intensive task. Each integration steps requires the retrieval of data from 4 time steps for the purposes of temporal interpolation and each particle requires between 64 and 512 data points from each time step for the purposes of spatial interpolation. Our implementations show good scalability with increased process count per database as shown in Figure 8. We performed two sets of experiments against the MHD dataset. For the first set we issued particle tracking requests for 100,000 particles over a time span of 0.5 (out of a total simulation time span of 2.56) with an integral time step of 0.001 (compared to a separation between simulation time steps of 0.0025 ). This corresponded to 1,000 integration rounds (500 pairs of predictor and corrector steps in the Runge-Kutta scheme) over 200 simulation time steps. The second set tracked 10,000 particles over a time span of 1.25 with an integral time step 0.0025 equal to the separation between the stored simulation time steps. This also corresponded to 1,000 integration rounds, but over 500 simulation time steps.

The task-parallel and data-parallel approaches show better parallel efficiency than synchronization at the mediator, which is to be expected as they allow each process to perform particle integration independently. For the first set of experiments, the small integration time step leads to slightly lower I/O time as database queries for sequences of a few integration steps request data from the same time steps, which makes the database cache very effective. For the second set of experiments even though the number of I/Os is the same every I/O has to bring data for at least one time step from disk as the integral time step is equal to the separation between stored simulation time steps. This increased I/O time reduces the parallel efficiency.

The task-parallel approach to particle tracking has parallel efficiency over $50 \%$ up to 8 processes per node (which is the number of cores available on each node). This shows that this approach is not sensitive to finer data-partitioning and can be effectively utilized if the data are distributed across larger database clusters. The reason why the speedup diminishes with increased process count is that the data reside in the same database tables and on the same set of disks and while computation scales with increased process count, I/O contention increases and we observe an overall sub-linear speedup. If the data are distributed across more database nodes we would expect to see even better speedup as the I/O would be performed to different databases.

\section{RELATED WORK}

Previous work on particle tracking and domain traversal approaches has focused on particle tracking for visualization of large datasets and in supercomputing environments. Ueng et al. 28 present a technique for out-of-core visualization of streamlines in large unstructured grids, which uses an octree to partition and restructure the raw data for fast retrieval. They focus on achieving optimal I/O performance with a small memory footprint. Yu et al. 30 describe a visualization technique that computes short integral segments called pathlets in time-varying vector fields. Their approach relies on a preprocessing step, which selects seed points and decomposes the data into blocks. This approach does not allow for user-specified seed points, which is important for the purposes of scientific analysis. Additionally, in our setting the data reside in relational databases and are used for a variety of analysis tasks in addition to particle tracking. Our goal is therefore to avoid preprocessing or restructuring the data.

Pugmire et al. 23 describe a hybrid approach that makes use of both static decomposition and loading data on demand. The process is coordinated by a master process that monitors load balance. Peterka et al. 21 investigate static and dynamic partitioning strategies and show that simple round-robin partitioning often outperforms dynamic partitioning. These approaches were demonstrated on up to 512 Cray XT cores and $32 \mathrm{~K}$ Blue Gene/P cores, respectively.

Kendall et al. 14 introduce a system called DStep aimed at simplifying domain traversal techniques. DStep utilizes different workers to perform domain traversal tasks. Workers are partitioned into groups with a communicator worker assigned to each group responsible for inter-group communication. The shift to group-based communication for domain traversal techniques is driven by the need to alleviate the network congestions induced by asynchronous communication. Our study also indicates that a purely data-parallel asynchronous approach may suffer performance penalties, however mostly due to its I/O pattern. In the DStep system 
the large distributed memory of a supercomputer is leveraged to perform all I/O upfront, which is not feasible for smaller analysis clusters.

Our work focuses on scientific analysis cluster environments, where large numerical simulation datasets are stored in a database cluster. The JHTDB store array data as a collection of binary large objects (BLOBs) in a relational database management system (Microsoft's SQL Server). The array manipulation tasks are performed by means of stored procedures or user-defined functions deployed in each database. There are only a few database systems that provide native support for array data and strive to handle large array datasets efficiently. These include RasDaMan 2], SciDB 26 and MonetDB/SciQL 18, 31. Similarly to the JHTDB, RasDaMan stores raster objects partitioned into tiles in a relational database system. SciDB is a database system build from the ground up with the goal of making arrays firstclass citizens. We expect that while I/O times may vary if a different database system was used to store the simulation data, the relative performance of the particle tracking methods studied is likely to remain the same

\section{CONCLUSIONS AND FUTURE WORK}

We have presented a study of particle tracking approaches, which advect particles for scientific analysis purposes in an open simulation laboratory, the JHTDB. Particle tracking is both data and computationally intensive task and the approaches that we compare are implemented with the goal of minimizing overall I/O. We examine data and task parallelization techniques as well as an approach that synchronizes execution at the mediator level. We evaluate these techniques on datasets 100s of TB in size in a live production environment. We observe that the task-parallel approach to particle tracking outperforms the other approaches by up to a factor of 3 for a variety of workloads and parameterizations. It achieves over $50 \%$ parallel efficiency and is able to scale the execution by performing the entire advection process on the database nodes of the analysis cluster environment.

Our study highlights the trade-offs between the batch execution of database queries for the retrieval of data necessary during each integration step and asynchronous processing and assignment of particles as they traverse the data volume and cross data boundaries. We find that the batch execution of particles, retrieving data from the same set of time steps as needed for temporal interpolation during each integration step outweighs the benefits of asynchronous processing. In order to support asynchronous processing the I/O pattern changes fundamentally and has to allow for the retrieval of data blocks from multiple different time steps, which results in slower I/O.

In the future, we plan to extend the analysis capabilities of the JHTDB and provide more built-in tools for the study of fluid dynamics in the simulation laboratory. One such capability in the context of particle tracking is the computation of the Finite-Time Lyapunov Exponent (FTLE) field. In recent years, significant efforts have been devoted to studying flow using visualizations of FTLEs 8, 9]. Nouanesengsy et al. 19. describe a framework and a parallelization strategy to trace the massive number of particles necessary for FTLE computation using a supercomputer. We plan to investigate similar techniques that can be adapted to the scientific analysis cluster environment.
Particle tracking is not yet available for one of the datasets recently added to the JHTDB, the channel flow dataset. This is because this dataset simulates turbulent flow between walls in a channel and the velocity goes to 0 near the walls. Our turbulence-research collaborators are still investigating the proper way to handle particles that approach the walls of the channel. We plan to extend the particle tracking capabilities of the JHTDB to the channel flow dataset in the near future.

We also plan to extend the JHTDB by providing a serverside computing environment for users similar to the CasJobs and MyDB services available in the Sloan Digital Sky Survery 15. In such an environment users can direct output of their batch queries to personal MyDB databases. In the case of particle tracking, this will allow users to store trajectories along with additional quantities at each particle position without having to transfer large amounts of data over the network. We also plan to provide server-side visualization and rendering capabilities that will allow users to visualize particle trajectories and other data in an interactive environment.

\section{ACKNOWLEDGMENTS}

The authors thank the Turbulence Database Group at Johns Hopkins University and four anonymous reviewers for their insightful comments and suggestions. This work, the software and hardware infrastructure of the simulation laboratory are supported in part by the National Science Foundation under Grants CMMI-0941530, ACI-1261715, OCI1244820 and AST-0939767 and Johns Hopkins University's Institute for Data Intensive Engineering \& Science.

\section{REFERENCES}

[1] H. Aluie, G. Eyink, E. Vishniac, S. Chen, K. Kanov, R. Burns, C. Meneveau, and A. Szalay. Forced MHD turbulence data set (2013). Available at http://turbulence.pha.jhu.edu/docs/READMEMHD.pdf.

[2] P. Baumann, A. Dehmel, P. Furtado, R. Ritsch, and N. Widmann. The Multidimensional Database System RasDaMan. In Proceedings of the 1998 ACM SIGMOD International Conference on Management of Data, SIGMOD '98, pages 575-577, 1998.

[3] D. Benveniste and T. Drivas. Asymptotic results for backwards two-particle dispersion in a turbulent flow. Physical Review E, 89(4):041003, 2014.

[4] DataScope. http://idies.jhu.edu/datascope.

[5] G. Eyink and D. Benveniste. Diffusion approximation in turbulent two-particle dispersion. Physical Review E, 88(4):041001, 2013.

[6] G. Eyink, E. Vishniac, C. Lalescu, H. Aluie, K. Kanov, K. Bürger, R. Burns, C. Meneveau, and A. Szalay. Flux-freezing breakdown in high-conductivity magnetohydrodynamic turbulence. Nature, 497(7450):466-9, 2013.

[7] K. Gustavsson, J. Einarsson, and B. Mehlig. Tumbling of small axisymmetric particles in random and turbulent flows. Physical Review Letters, 112(1):014501, 2014.

[8] G. Haller. Finding finite-time invariant manifolds in two-dimensional velocity fields. Chaos: $A n$ 
Interdisciplinary Journal of Nonlinear Science, 10(1):99-108, 2000.

[9] Haller, G. Distinguished material surfaces and coherent structures in three-dimensional fluid flows. Physica D, 149(4):248-277, 2001.

[10] A. J. G. Hey, S. Tansley, and K. M. Tolle. The Fourth Paradigm: Data-Intensive Scientific Discovery. Microsoft Research, 2009.

[11] M. Holzner, A. Liberzon, N. Nikitin, B. Lüthi, W. Kinzelbach, and A. Tsinober. A lagrangian investigation of the small-scale features of turbulent entrainment through particle tracking and direct numerical simulation. Journal of Fluid Mechanics, 598:465-475, 32008.

[12] J. Jucha, H. Xu, A. Pumir, and E. Bodenschatz. Time-reversal-symmetry breaking in turbulence. Physical Review Letters, 113(5):054501, 2014.

[13] K. Kanov, E. Perlman, R. Burns, Y. Ahmad, and A. Szalay. I/O Streaming Evaluation of Batch Queries for Data-intensive Computational Turbulence. In Proceedings of 2011 International Conference for High Performance Computing, Networking, Storage and Analysis, SC '11, pages 29:1-29:10, 2011.

[14] W. Kendall, J. Wang, M. Allen, T. Peterka, J. Huang, and D. Erickson. Simplified parallel domain traversal. In Proceedings of 2011 International Conference for High Performance Computing, Networking, Storage and Analysis, SC '11, pages 10:1-10:11, 2011.

[15] N. Li and A. R. Thakar. CasJobs and MyDB: A Batch Query Workbench. Computing in Science and Engineering, 10(1):18-29, 2008.

[16] Y. Li, E. Perlman, M. Wan, Y. Yang, C. Meneveau, R. Burns, S. Chen, A. Szalay, and G. Eyink. A public turbulence database cluster and applications to study Lagrangian evolution of velocity increments in turbulence. Journal of Turbulence, 9:N31, 2008.

[17] D. Livescu, C. Canada, K. Kanov, Burns, R. \& IDIES staff, and J. Pulido. Homogeneous Buoyancy driven turbulence data set (2014). Available at http://turbulence.pha.jhu.edu/docs/READMEHBDT.pdf.

[18] MonetDB. http://monetdb.cwi.nl/.

[19] B. Nouanesengsy, T.-Y. Lee, K. Lu, H.-W. Shen, and T. Peterka. Parallel particle advection and FTLE computation for time-varying flow fields. In Proceedings of the International Conference on High Performance Computing, Networking, Storage and Analysis, SC '12, pages 61:1-61:11, 2012.

[20] E. Perlman, R. Burns, Y. Li, and C. Meneveau. Data Exploration of Turbulence Simulations Using a Database Cluster. In Proceedings of the 2007 ACM/IEEE Conference on Supercomputing, SC '07, pages 23:1-23:11, 2007.

[21] T. Peterka, R. Ross, B. Nouanesengsy, T.-Y. Lee, H.-W. Shen, W. Kendall, and J. Huang. A study of parallel particle tracing for steady-state and time-varying flow fields. In Proceedings of the 2011 IEEE International Parallel \& Distributed Processing Symposium, IPDPS '11, pages 580-591, 2011.

[22] S. B. Pope. Lagrangian pdf methods for turbulent flows. Annual Review of Fluid Mechanics, 26(1):23-63, 1994.
[23] D. Pugmire, H. Childs, C. Garth, S. Ahern, and G. H. Weber. Scalable computation of streamlines on very large datasets. In Proceedings of the Conference on High Performance Computing Networking, Storage and Analysis, SC '09, pages 16:1-16:12, 2009.

[24] M. Raiola, S. Discetti, and A. Ianiro. On piv random error minimization with optimal pod-based low-order reconstruction. Experiments in Fluids, 56(4), 2015.

[25] The Sloan Digital Sky Survey. http://www.sdss.org/.

[26] M. Stonebraker, J. Becla, D. J. DeWitt, K. Lim, D. Maier, O. Ratzesberger, and S. B. Zdonik. Requirements for Science Data Bases and SciDB. In Proceedings of the Fourth Biennial Conference on Innovative Data Systems Research, CIDR '09, 2009.

[27] A. S. Szalay, G. Bell, J. Vandenberg, A. Wonders, R. Burns, D. Fay, J. Heasley, T. Hey, M. Nieto-Santisteban, A. Thakar, C. van Ingen, and R. Wilton. GrayWulf: Scalable Clustered Architecture for Data Intensive Computing. In Proceedings of the 42nd Hawaii International Conference on System Sciences, HICSS '09, pages 1-10, 2009.

[28] S.-K. Ueng, C. Sikorski, and K.-L. Ma. Out-of-core streamline visualization on large unstructured meshes. IEEE Transactions on Visualization and Computer Graphics, 3(4):370-380, 1997.

[29] H. Yu, K. Kanov, E. Perlman, J. Graham, E. Frederix, R. Burns, A. Szalay, G. Eyink, and C. Meneveau. Studying lagrangian dynamics of turbulence using on-demand fluid particle tracking in a public turbulence database. Journal of Turbulence, 13:N12, 2012.

[30] H. Yu, C. Wang, and K.-L. Ma. Parallel hierarchical visualization of large time-varying $3 \mathrm{~d}$ vector fields. In Proceedings of the 2007 ACM/IEEE Conference on Supercomputing, SC '07, pages 24:1-24:12, 2007.

[31] Y. Zhang, M. Kersten, M. Ivanova, and N. Nes. SciQL: Bridging the Gap Between Science and Relational DBMS. In Proceedings of the 15th Symposium on International Database Engineering 85 Applications, IDEAS '11, pages 124-133, 2011. 